\title{
Detection of strains of endophytic bacteria of the genus Bacillus with the most pronounced growth-stimulating and protective properties
}

Cherepanova E.A., Veselova S.V., Alekseev V.Yu., Maksimov I.V.

Institute of Biochemistry and Genetics of the Ufa Federal Research Centre

of the Russian Academy of Sciences, Ufa, Russia.

E-mail: k_cherepanova@mail.ru

Key message. Treatment of wheat with endophytic bacteria increases plant growth and reduces the area of leaf infection, but the degree of manifestation of these properties depends on the bacterial strain.

Keywords: wheat, Bacillus, resistance, Stagonospora nodorum

Agriculture is increasingly using the ability of bacteria to directly inhibit the growth of phytopathogens, penetrate plant tissues without harming the latter and stimulate both growth and plant resistance to biotic and abiotic environmental factors. In this connection, the search for the most effective for plant growth and development of bacterial strains of wheat endosymbionts and study their ability to protect plants from pathogens is relevant.

The aim of the study was to study the influence of endophytic bacterial strains from the laboratory collection on germination energy and germination of seeds, growth of raw and dry biomass, as well as on the resistance of wheat to the causative agent of Septoria nodorum blotch - Stagonospora nodorum Berk. fungi.

Strains of Bacillus genus bacteria with pronounced fungistatic properties were selected for experiments from the laboratory collection: $B$. subtilis 26D (collection ARRIAM St.-Pb. Pushkin, №128), B. subtilis 11VM (ARRIAM №519), B. thuringiensis B-6066 (Russian National Collection of Industrial Microorganisms), and isolates bacteria B. subtilis Tas1 and $B$. subtilis Tas8-2, isolated from wheat, zoned in the Republic of Bashkortostan. In order to assess the impact of bacteria on wheat resistance to the causative agent of Septoria nodorum blotch, 7-day wheat germ leaves were inoculated with St. nodorum spores from the collection of the Plant Immunity Biochemistry Laboratory of IBG RAS were used.

Efficient concentrations of bacterial spore were selected to treat wheat seeds. It is interesting to note that effective concentrations of $B$. subtilis $11 \mathrm{BM}$ and $B$. subtilis Tas8-2 were twice lower than in other strains. Concentrations below effective concentrations were weaker in plant growth, and higher concentrations could even inhibit them. Treatment of seeds with bacterial strains and isolates in optimal concentrations increased seed germination by about 30-40\% of the control level. The greatest effect was given by strain $B$. subtilis 26D. The strain of B. subtilis $11 \mathrm{BM}$ and $B$. subtilis Tas 1 were slightly weaker in stimulating seed germination. The treatment of wheat seeds with suspensions of bacterial spores contributed to the increase in the mass of wheat germplants, while the treatment of B. subtilis 26D, B. subtilis Tas1 and B. thuringiensis B-6066 had the strongest effect - in these germplants the dry mass exceeded the mass of untreated bacterial samples by $32 \%$.

During treatment of $B$. subtilis $11 \mathrm{BM}$ and Tas 1 the most expressed resistance to the causative agent of Septoria nodorum blotch was shown, which was expressed in 4 times less area of wheat leaf infection in comparison with untreated control. Leaves treated with B. thuringiensis B-6066 showed the least resistance to St. nodorum. However, even with the last of these strains, the leaf infection area was twice less than that of untreated samples. Summarizing the data obtained, it can be concluded that the B. subtilis Tas1 isolate had the most pronounced both stimulating plant growth and sufficiently high protective properties against the pathogenic fungus.

The results of this work can have practical value and help to create environmentally friendly drugs that combine both growthstimulating and increasing resistance to phytopathogens.

The research has been carried out using equipment from the Regional analytical Centre of collective usage "Agidel" and the

Unique scientific installation "Kodink" with partial financial support of the Russian Foundation for Basic Research (RFBR) grant № 17-29-08014 (2018). 